\title{
Algebraic Implications of Composability of Physical Systems
}

\author{
Emil Grgin and Aage Petersen \\ Belfer Graduate School of Science, Yeshiva University, New York, N.Y. 10033, USA
}

\begin{abstract}
From classical and quantum mechanics we abstract the concept of a two-product algebra. One of its products is left unspecified; the other is a Lie product and a derivation with respect to the first. From composition of physical systems we abstract the concept of composition classes of such two-product algebras, each class being a semigroup with a unit. We show that the requirement of mutual consistency of the algebraic and the semigroup structures completely determines both the composition classes and the twoproduct algebras they consist of. The solutions are labelled by a single parameter which in the physical case is proportional to the square of the quantum of action.
\end{abstract}

\section{Introduction}

In both classical and quantum mechanics, the set of physical variables belonging to a system with a given number of degrees of freedom is a linear space with two algebraic products. The classical variables are real-valued functions on a phase space, and the two products are the usual multiplication and the Poisson bracket of such functions. The quantal variables are self-adjoint operators on a Hilbert space, and the two products are $\frac{1}{2}[,]_{+}$and $(i \hbar)^{-1}[,]_{-}$, where $[,]_{+}$and $[,]_{-}$ denote the anticommutator and the commutator of such operators. Multiplication of classical functions is commutative and associative, while the anticommutator of quantal operators is a commutative but not associative product. Both the Poisson bracket and the commutator are Lie products. Further, in both mechanics the two products are related by the same "distribution law", i.e. the derivation rule. For example, if $f, g, h$ are classical variables and $\cdot$ and $\{$,$\} denote$ the two classical products, then $\{f, g \cdot h\}=\{f, g\} \cdot h+g \cdot\{f, h\}$.

From the examples provided by classical and quantum mechanics we abstract the concept of a two-product algebra $\{\mathscr{H}, \tau, \alpha\}$. In the definition of this structure, the properties of the product $\tau$ are left unspecified, while the product $\alpha$ is required to be a Lie product and the operators $f \alpha$, with $f \in \mathscr{H}$, are to be derivations with respect to the product $\tau$. 
When two classical or two quantal systems interact, they form a single classical or quantal system. The set of physical variables belonging to the composite system is the tensor product of the component spaces of physical variables, and the composite space carries a two-product structure which is induced by the component two-product structures.

From the manner in which physical systems compose in classical and quantum mechanics we abstract the concept of a composition class $\mathscr{J}$ of two-product algebras. The structure $\mathscr{J}$ is defined to be a semigroup with a unit.

Our purpose is to investigate the restrictions which the semigroup structure of a composition class imposes on the algebraic structure of the two-product objects that belong to the class [1].

In Section II we determine the composition classes of two-product algebras and the corresponding composition laws for the products $\tau$ and $\alpha$. We show that all composition classes are obtained from a family $\left\{\mathscr{J}_{a}\right\}$ whose members are labelled by a parameter $a$ which is defined modulo the squares in the field of scalars, $\mathscr{F}$. When $\mathscr{I}$ is the field $\mathbb{R}$ of real numbers and $a>0, a$ is proportional to the square of the quantum of action.

The composition class $\mathscr{I}_{0}$, with $a=0$, is called classical, and the classes $\mathscr{I}_{a}$, with $a \neq 0$, are called quantal. The class parameter $a$ also occurs as an element of structure of the individual two-product algebras in the class. In all algebras in a class $\mathscr{I}_{a}$, with $a$ arbitrary, the product $\tau$ is symmetric and denoted by $\sigma$. In the classical case, the substructure $\{\mathscr{H}, \sigma\}$ of the two-product algebra $\{\mathscr{H}, \sigma, \alpha\}$ is a commutative associative algebra, whereas in the quantal case $\{\mathscr{H}, \sigma\}$ is a special Jordan algebra.

All algebras $\{\mathscr{H}, \sigma, \alpha\}$ have an "associative envelope", i.e. a single-product associative algebra $\{\mathscr{\mathscr { H }}, \beta\}$, such that $\beta=\sigma+b \alpha$, where $b=\sqrt{-a}$ if $a \neq 0$ and $b^{2}=0$ if $a=0$. When $a \geqq 0, \beta$ is not a product in $\mathscr{H}$ itself but in a space $\tilde{\mathscr{H}}$ over an extension of the field $\mathscr{F}$. In the quantal case $(a>0)$, this extension is the field $\mathscr{F}[\sqrt{-a}](=\mathbb{C}$ for $\mathscr{F}=\mathbb{R})$, whereas in the classical case $(a=0)$, the extension is the ring $\mathscr{F} \oplus b \mathscr{F}$.

In Appendix A we discuss briefly the composition of identical two-product algebras. We show that the symmetric tensor powers of a two-product algebra form a composition class, whereas the exterior powers do not. However, the direct sum of a symmetric power and an antisymmetric power (of the same degree) is a two-product algebra, and a composition class can be formed of such algebras.

Finally, in Appendix B we show that the composition laws for the products in a quantal algebra lead to a simple proof of a result similar to C. L. Mehta's theorem [2] on the relation between the classical and quantal Lie products.

\section{Composition of Two-Product Algebras}

A two-product algebra $\mathfrak{U}=\{\mathscr{H}, \tau, \alpha\}$ is a linear space $\mathscr{H}$ over a (commutative) field $\mathscr{F}$, equipped with two products, $\tau, \alpha: \mathscr{H} \otimes \mathscr{H} \rightarrow \mathscr{H}$, which satisfy the identities:

$$
\begin{aligned}
& f \alpha g=-g \alpha f \\
& (f \alpha g) \alpha h+(g \alpha h) \alpha f+(h \alpha f) \alpha g=0 \\
& f \alpha(g \tau h)=(f \alpha g) \tau h+g \tau(f \alpha h)=0
\end{aligned}
$$

for all $f, g, h \in \mathscr{H}$. 
If $\mathscr{H}$ is a topological linear space and the products $\tau, \alpha$ are required to be continuous, the relations (1), (2), (3) define a topological two-product algebra. In this paper we consider only purely algebraic questions.

A composition class is a set $\mathscr{J}$ of two-product algebras, equipped with a product $\bigcirc: \mathscr{J} \times \mathscr{J} \rightarrow \mathscr{J}$ with the following properties:

1) If $\mathfrak{A}_{1}, \mathfrak{U}_{2} \in \mathscr{J}$ and $\mathfrak{U}_{12}=\mathfrak{U}_{1} \bigcirc \mathfrak{U}_{2}$. then $\mathscr{H}_{12}=\mathscr{H}_{1} \otimes \mathscr{H}_{2}$,

i.e. the underlying linear space of the product algebra is the tensor product of the underlying linear spaces of the component algebras.

2) Associativity:

$\left(\mathfrak{U}_{1} \bigcirc \mathfrak{U}_{2}\right) \bigcirc \mathfrak{U}_{3}=\mathfrak{U}_{1} \bigcirc\left(\mathfrak{U}_{2} \bigcirc \mathfrak{U}_{3}\right)$.

3) Existence of a unit.

The field $\mathscr{F}$, considered as a two-product algebra $\{\mathscr{F}, \tau, \alpha\}$, where $\tau$ is the product in $\mathscr{F}$ and $\alpha=0$, is a unit for the composition product:

$\mathscr{F} \bigcirc \mathfrak{U}=\mathfrak{U}=\mathfrak{U} \bigcirc \mathscr{F}$

for all $\mathfrak{U} \in \mathscr{J}$.

With these conditions on the composition product $\bigcirc$, a composition class $\mathscr{J}$ of two-product algebras is a semigroup with a unit.

A two-product algebra $\{\mathscr{H}, \tau, \alpha\}$ in which $(f \alpha g) \tau h=0$ for all $f, g, h \in \mathscr{H}$ shall be called trivial. We do not consider composition classes which consist exclusively of trivial algebras.

Let $\mathfrak{U}_{1}$ and $\mathfrak{U}_{2}$ be a pair of non-trivial algebras belonging to a composition class $\mathscr{J}$. We shall determine the products $\tau_{12}, \alpha_{12}$ in the composite algebra $\mathfrak{U}_{12}=\mathfrak{A}_{1} \bigcirc \mathfrak{A}_{2}$ induced by the component products $\tau_{1}, \alpha_{1}, \tau_{2}, \alpha_{2}$ and consistent with the conditions (1)-(6).

For this purpose it is convenient to decompose the products $\tau$ into a symmetric and an antisymmetric part, i.e. $\tau=\sigma+\pi, f \sigma g=\frac{1}{2}(f \tau g+g \tau f), f \pi g=\frac{1}{2}(f \tau g-g \tau f)$. With this decomposition, and in view of relation (1), all products, $\sigma, \pi, \alpha$ have definite symmetry properties.

A general element of $\mathscr{H}_{12}$ is a linear combination of decomposable elements $f_{1} \otimes f_{2}$, where $f_{1} \in \mathscr{H}_{1}$, and $f_{2} \in \mathscr{H}_{2}$, but since $\sigma_{12}, \pi_{12}$, and $\alpha_{12}$ are linear mappings it suffices to consider their effect on the decomposable elements themselves. For typographical economy, tensor product symbols shall be omitted, i.e. $f_{1} \otimes f_{2} \equiv f_{1} f_{2}$. Further, expressions of the form $(f \varrho g)_{i}$, where $i=1,2, \ldots$ and $\varrho=\sigma, \pi, \alpha$, are to be interpreted as $f_{i} \varrho_{i} g_{i} \in \mathscr{H}_{i}$.

The most general antisymmetric algebraic product that can be formed from the component products is

$$
\begin{aligned}
& \left(f_{1} f_{2}\right) \alpha_{12}\left(g_{1} g_{2}\right) \\
& \quad=m(f \sigma g)_{1}(f \alpha g)_{2}+n(f \alpha g)_{1}(f \sigma g)_{2}+p(f \sigma g)_{1}(f \pi g)_{2}+q(f \pi g)_{1}(f \sigma g)_{2} .
\end{aligned}
$$

Similarly, omitting the obvious variables,

$$
\pi_{12}=c \sigma_{1} \pi_{2}+d \pi_{1} \sigma_{2}+x \sigma_{1} \alpha_{2}+y \alpha_{1} \sigma_{2} .
$$

The most general symmetric product is of the form

$$
\sigma_{12}=r \sigma_{1} \sigma_{2}+s \alpha_{1} \alpha_{2}+u \alpha_{1} \pi_{2}+v \pi_{1} \alpha_{2}+w \pi_{1} \pi_{2} .
$$

In these expressions the coefficients $m, n, \ldots w$ are elements of the field $\mathscr{F}$. 
The symmetric product $\sigma$ occurs in all terms of the antisymmetric products $\alpha_{12}$ and $\pi_{12}$ and hence it cannot vanish if the algebras are composable. In other words, the producr $\tau$ cannot be antisymmetric. An obvious solution for the product $\pi$ is a multiple of $\alpha$.

The requirement that the field $\mathscr{F}$ be a unit for the composition product imposes strong restrictions on the coefficients in the expressions (7). Relation (6), together with the choice $\mathfrak{U}_{1}=\mathscr{F}$ and $f_{1}=g_{1}=1 \in \mathscr{F}$ in Equation (7a), yield $m=1$ and $p=0$. Similarly, $\mathfrak{U}_{2}=\mathscr{F}, f_{2}=g_{2}=1 \in \mathscr{F}$ gives $n=1, q=0$. The same arguments applied to the products $\pi$ and $\sigma$ yield $c=d=r=1$ and $x=y=0$.

Further restrictions are imposed on the remaining coefficients in expression (7c) by the requirement that the composition product be associative. Equation (5) applied to the product $\alpha_{123}$ in $\mathfrak{U}_{123}=\mathfrak{U}_{1} \bigcirc \mathfrak{A}_{2} \bigcirc \mathfrak{U}_{3}$ reads

$$
\alpha_{123}=\alpha_{(12) 3}=\alpha_{1(23)} \text {. }
$$

Substitution of relations (7a) and (7c) shows that the coefficients $u, v$, and $w$ must vanish. Hence, the composition laws reduce to

$$
\begin{aligned}
& \alpha_{12}=\sigma_{1} \alpha_{2}+\alpha_{1} \sigma_{2} \\
& \pi_{12}=\sigma_{1} \pi_{2}+\pi_{1} \sigma_{2} \\
& \sigma_{12}=\sigma_{1} \sigma_{2}+s \alpha_{1} \alpha_{2} .
\end{aligned}
$$

The associativity condition (5) applied to the product $\sigma$, i.e. $\sigma_{1(23)}=\sigma_{(12) 3}$, leaves the coefficient $s$ arbitrary, while the condition $\pi_{1(23)}=\pi_{(12) 3}$ yields $s=0$. Hence, if $s \neq 0$ the associativity condition for the composition product $\bigcirc$ does not allow the algebraic product $\tau$ in $\{\mathscr{H}, \tau, \alpha\} \varepsilon \mathscr{I}$ to have an antisymmetric part which is different from the product $\alpha$.

So far we have derived the conditions which are imposed on the composition laws for the algebraic products $\alpha, \pi$, and $\sigma$ by the assumption that a composition class be a semigroup with a unit. We shall now investigate how these conditions interact with the identities defining the individual algebras in a composition class (the Jacobi identity and the derivation rule).

Using relation (8a) one sees that the generic term in the Jacobi identity (2) is

$$
\begin{aligned}
& \left(f_{1} f_{2}\right) \alpha_{12}\left(\left(g_{1} g_{2}\right) \alpha_{12}\left(h_{1} h_{2}\right)\right) \\
& =(f \sigma(g \sigma h))_{1}(f \alpha(g \alpha h))_{2}+(f \sigma(g \alpha h))_{1}(f \alpha(g \sigma h))_{2} \\
& \quad+(f \alpha(g \sigma h))_{1}(f \sigma(g \alpha h))_{2}+(f \alpha(g \alpha h))_{1}(f \sigma(g \sigma h))_{2} .
\end{aligned}
$$

The Jacobi identity then reads

$$
\sum_{\text {cycl. }}\left\{(f \sigma(g \sigma h))_{1}(f \alpha(g \alpha h))_{2}+(f \alpha(g \alpha h))_{1}(f \sigma(g \sigma h))_{2}\right\}=0 .
$$

Addition of this identity to the one obtained by interchange of $g_{1}$ and $h_{1}$ yields the relation

$$
\left\{[f, g, h]_{1}+[f, h, g]_{1}\right\}(f \alpha(g \alpha h))_{2}=\left\{(g \alpha(h \alpha f))_{1}+(h \alpha(g \alpha f))_{1}\right\}[h, f, g]_{2}
$$


where the symbol $[,$,$] denotes the \sigma$-associator, i.e. $[f, g, h] \equiv(f \sigma g) \sigma k-f \sigma(g \sigma h)$. Relation (10) implies proportionality of the associator and the double $\alpha$-product:

$$
a_{i}(f \alpha(g \alpha h))_{i}=b_{i}[h, f, g]_{i}
$$

where $i=1,2$ and the coefficients $a_{i}, b_{i}$, which cannot both vanish, are defined up to a proportionality factor. Substitution of relation (11) into relation (10) yields

$$
a_{1} b_{2}=b_{1} a_{2} .
$$

One sees that $b_{1}=0$ implies $b_{2}=0$. Hence, if a composition class contains an algebra with $b=0$, then all algebras in the composition class have $b=0$.

According to relation (11), $b=0$ implies that $f \alpha(g \alpha h)=0$ for all algebras in the composition class. Applied to relation (9), this conclusion yields the identity

$$
(f \sigma(g \alpha h))_{1}(f \alpha(g \sigma h))_{2}+(f \alpha(g \sigma h))_{1}(f \sigma(g \alpha h))_{2}=0 .
$$

By taking $g_{2}=h_{2}$ one gets

$$
(f \sigma(g \alpha h))_{1}=0 \text { or }(f \alpha(g \sigma g))_{2}=0 .
$$

Substitution of $g+h$ for $g$ in the second equation gives $(f \alpha(g \sigma h))_{2}=0$. Hence, in both cases the algebras are trivial. Consequently, all algebras in a composition class with $b=0$ are trivial. Thus, we are not interested in this case.

If a composition class contains an algebra with $b \neq 0$, then all $\mathfrak{U}_{i}$ in the composition class have $b_{i} \neq 0$, and since $a_{i}$ and $b_{i}$ are defined only up to a common factor we can choose $b_{i}=1$. Relation (12) then reads $a_{1}=a_{2}$, i.e. the constant $a$ is the same for all algebras in the composition class, and relation (11) becomes

$$
[h, f, g]=a f \alpha(g \alpha h) \text {. }
$$

Next, we consider the derivation condition (3) for the composite $\alpha$ and $\sigma$ products:

$$
\begin{aligned}
\left(f_{1} f_{2}\right) \alpha_{12}\left\{\left(g_{1} g_{2}\right) \sigma_{12}\left(h_{1} h_{2}\right)\right\}= & \left\{\left(f_{1} f_{2}\right) \alpha_{12}\left(g_{1} g_{2}\right)\right\} \sigma_{12}\left(h_{1} h_{2}\right) \\
& +\left(g_{1} g_{2}\right) \sigma_{12}\left\{\left(f_{1} f_{2}\right) \alpha_{12}\left(h_{1} h_{2}\right)\right\} .
\end{aligned}
$$

Use of the composition laws (8a), (8c), and the Jacobi identity for the component $\alpha$-products yield the relation

$$
\begin{aligned}
& ((f \sigma g) \sigma h)_{1}((f \alpha g) \sigma h)_{2}+(g \sigma(f \sigma h))_{1}(g \sigma(f \alpha h))_{2} \\
& \quad-(f \sigma(g \sigma h))_{1}(f \alpha(g \sigma h))_{2}+((f \alpha g) \sigma h)_{1}((f \sigma g) \sigma h)_{2} \\
& \quad+(g \sigma(f \alpha h))_{1}(g \sigma(f \sigma h))_{2}-(f \alpha(g \sigma h))_{1}(f \sigma(g \sigma h))_{2} \\
& \quad+s\left\{((f \alpha h) \sigma g)_{1}((f \alpha g) \alpha h)_{2}+((g \alpha f) \sigma h)_{1}(g \alpha(f \alpha h))_{2}\right. \\
& \left.\quad+((f \alpha g) \alpha h)_{1}((f \alpha h) \sigma g)_{2}+(g \alpha(f \alpha h))_{1}((g \alpha f) \sigma h)_{2}\right\}=0 .
\end{aligned}
$$

Addition of this identity to the one obtained by interchange of $f_{1}$ and $g_{1}$, and use of the derivation rule for the component products give the relation

$$
\begin{gathered}
\{[f, g, h]+[g, f, h]+g \alpha(h \alpha f)+f \alpha(h \alpha g)\}_{1}((f \alpha g) \sigma h)_{2} \\
=-((f \sigma g) \alpha h)_{1}\{[f, h, g]+\operatorname{sh} \alpha(g \alpha f)\}_{2}
\end{gathered}
$$


which implies

$$
c_{i}(h \sigma(g \alpha f))_{i}=d_{i}\{[f, h, g]+\operatorname{sh} \alpha(g \alpha f)\}_{i}
$$

where $c_{i}$ and $d_{i}$ are scalars which do not both vanish. If both $c_{i}$ and $d_{i}$ are different from zero, then substitution of the double $\alpha$-product for the associator according to relation (13) shows that $h \sigma(g \alpha f)$ is proportional to $h \alpha(g \alpha f)$. Since these expressions have different symmetry properties they must both vanish, i.e. the algebra is trivial. The same result holds if $d_{i}=0$. Consequently, if the algebra is to be non-trivial, the coefficient $c_{i}$ must vanish, and this yields the identity

$$
[f, h, g]_{i}+s(h \alpha(g \alpha f))_{i}=0 .
$$

Comparison with relation (13) shows that

$$
a=-s \text {. }
$$

While the results obtained previously from the semigroup structure of a composition class were restrictions on the class parameters in the composition laws for the algebraic products, the result $s=-a$ relates the semigroup product $\bigcirc$ to the associativity properties of the algebraic product $\sigma$ in the individual algebras belonging to the composition class.

The closure of a composition class under the product $\bigcirc$ implies that the association constant $a$ of a composite algebra must be the same as that of the component algebras. It is straightforward to verify that if the equation

$$
[f, g, h]_{i}=a(g \alpha(h \alpha f))_{i}
$$

is true for $i=1,2$, and if $\alpha_{12}$ and $\sigma_{12}$ are given by relations (8a), (8c), then this equation is also true for $i=12$. Hence, the closure condition imposes no restrictions on the constant $a$.

It remains to investigate the restrictions imposed by the derivation condition for the antisymmetric part $\pi$ of the product $\tau$. Since $\pi \neq \alpha$ is impossible in composition classes where $s \neq 0$, it suffices to consider the case $s=0$, in which the algebras $\{\mathscr{H}, \sigma\}$ are associative. With this condition, the derivation rule (3) for the product $\alpha_{12}$ with respect to the product $\pi_{12}$ reduces to

$$
\begin{aligned}
& \{f \sigma(g \pi h)-(f \sigma g) \pi h\}_{1}((f \alpha g) \sigma h)_{2}+\{f \sigma(g \pi h)-g \pi(f \sigma h)\}_{1}(g \sigma(f \alpha h))_{2} \\
& \quad+((f \alpha g) \sigma h)_{1}\{f \sigma(g \pi h)-(f \sigma g) \pi h\}_{2}+(g \sigma(f \alpha h))_{1}\{f \sigma(g \pi h)-g \pi(f \sigma h)\}_{2}=0 .
\end{aligned}
$$

Addition of this identity to the one obtained by interchange of $g_{1}$ and $h_{1}$ yields

$$
b_{i}(f \alpha(g \sigma h))_{i}=\{g \pi(f \sigma h)+h \pi(f \sigma h)\}_{i}
$$

where $b_{i}$ is a scalar. By taking the cyclic sum of both sides of this equation one obtains a new equation in which the left side vanishes identically. Hence,

$$
b_{i}(f \alpha(g \sigma h))_{i}=-(f \pi(g \sigma h))_{i}
$$

from which one concludes that $\pi$ is proportional to $\alpha$ if $g \sigma h$ is an arbitrary element of $\mathscr{H}$. If necessary, this condition can be satisfied by adjunction of a unit to the commutative associative algebra $\{\mathscr{H}, \sigma\}$. 
Thus, we have the result that the product $\tau$ in an algebra $\{\mathscr{H}, \tau, \alpha\}$ is of the form $\tau=\sigma+c \alpha$, where $c$ is a scalar. Further, the easily verified identity

$$
[f, g, h]_{\tau}=[f, g, h]_{\sigma}+c^{2}[f, g, h]_{\alpha}
$$

together with Equation (13) and the Jacobi identity (2) yield the association relation

$$
[f, g, h]_{\tau}=\left(a+c^{2}\right)[f, g, h]_{\alpha} .
$$

Relation (17) shows that the product $\tau$ satisfies the Jordan identity $\left[f^{2}, g, f\right]_{\tau}=0$ and the flexible law $[f, g, f]_{\tau}=0$. Thus, in an algebra $\{\mathscr{H}, \tau, \alpha\}$, the substructure $\{\mathscr{H}, \tau\}$ is a non-commutative Jordan algebra [3].

Substitution of $\sigma=\tau-c \alpha$ into relations (8a) and (8c) and use of Equation (15) yields the composition laws for the products $\alpha$ and $\tau$ in algebras $\{\mathscr{H}, \tau, \alpha\}$ :

$$
\begin{aligned}
& \alpha_{12}=\tau_{1} \alpha_{2}+\alpha_{1} \tau_{2}-2 c \alpha_{1} \alpha_{2}, \\
& \tau_{12}=\tau_{1} \tau_{2}-\left(a+c^{2}\right) \alpha_{1} \alpha_{2} .
\end{aligned}
$$

Thus, the composition classes $\mathscr{J}$ are labelled by the parameters $a$ and $c$. The classes for which $a=0$ shall be called classical and the classes with $a \neq 0$ shall be called quantal. Since relation (13) is automatically satisfied for algebras $\{\mathscr{H}, \sigma, \alpha\}$ in which both $[f, g, h]_{\sigma}=0$ and $[f, g, h]_{\alpha}=0$, such algebras belong to both the classical and the quantal composition classes.

The system of identities (1), (2), (3), (17) defining an algebra $\{\mathscr{H}, \tau, \alpha, c, a\}$ with parameters $c$ and $a$ is invariant with respect to the group of transformations

$$
T:\left[\begin{array}{c}
\mathscr{H} \\
\tau \\
\alpha \\
c \\
a
\end{array}\right] \rightarrow\left[\begin{array}{c}
\mathscr{H} \\
\tau \\
t \alpha \\
t^{-1} c \\
t^{-2} a
\end{array}\right]
$$

where $t$ is a non-zero scalar. The orbits of this group are the isomorphism classes of the composition classes $\mathscr{J}$. For algebras $\{\mathscr{H}, \sigma, \alpha, 0, a\}$ the orbits of the parameter $a$ are the sets $\mathscr{F} /$ sq $\mathscr{F}$, where sq $\mathscr{F}$ denotes the squares in the field $\mathscr{F}$. The fixed point $\{0\}$ corresponds to the classical algebras $\{\mathscr{H}, \sigma, \alpha\}$, and the other cosets of sq $\mathscr{F}$ correspond to the composition classes of quantal algebras. In the case $\mathscr{F}=\mathbb{R}$, there are two quantal composition classes, $\mathscr{J}_{+}$and $\mathscr{J}_{-}$.

If $\{\mathscr{H}, \sigma, \alpha\}$ is a quantal algebra with a parameter $a$ such that $-a$ is a square in the field $\mathscr{F}$, then relation (17) shows that the product $\beta=\sigma+\sqrt{-a} \alpha$ in $\mathscr{H}$ is associative. If $-a$ is not a square, then $\beta$ is a product in the space $\tilde{\mathscr{H}}=\mathscr{H} \oplus \sqrt{-a} \mathscr{H}$. [This is the case in quantum mechanics, where $\mathscr{F}=\mathbb{R}$ and $a=(\hbar / 2)^{2}$.] Consequently, any quantal algebra $\{\mathscr{H}, \sigma, \alpha\}$ has an associative envelope $\{\mathscr{H}, \beta\}$ or $\{\tilde{\mathscr{H}}, \beta\}$. With respect to $\beta$, the products $\sigma$ and $\alpha$ are $\sigma=\frac{1}{2}[,]_{+}, \alpha=(1 / 2 \sqrt{-a})[,]_{-}$ or the restrictions of the anticommutator and the commutator to the space $\mathscr{H}$. It follows that in a quantal algebra $\{\mathscr{H}, \sigma, \alpha\}$, the substructure $\{\mathscr{H}, \sigma\}$ is a special Jordan algebra. 
If $\{\mathscr{H}, \sigma, \alpha\}$ is a classical algebra and $\beta=\sigma+b \alpha$, relation (16) shows that $\beta$ is associative if $b^{2}=0$. Thus, in order to obtain an associative envelope of a classical algebra, a nilpotent element, $b$, must be adjoined to the field.

One sees that in the classical case $(a=0)$ and in the quantal case with - a not a square in $\mathscr{F}$ (to which quantum mechanics belong), obtaining the associative envelope requires an extension of the field of scalars. However, while the quantal case requires an extension of $\mathscr{F}$ to a new structure $\mathscr{F}[\sqrt{-a}]$, which is also a field, the classical case requires an extension of $\mathscr{F}$ to a new structure $\mathscr{F} \oplus b \mathscr{F}$, with $b^{2}=0$, which is a ring. Hence, the underlying set of a classical $\beta$-algebra is not a linear space, but a module over the ring $\mathscr{F} \oplus b \mathscr{F}$.

If $\mathscr{F}$ is the field $\mathbb{R}$ of real numbers, one can subsume the two cases by means of the $2 \times 2$ real matrix

$$
b=\left(\begin{array}{cc}
0 & 2 \cosh ^{2} \omega / 2 \\
-2 \sinh ^{2} \omega / 2 & 0
\end{array}\right)
$$

which yields $b^{2}=-\sinh ^{2} \omega \cdot I$, where $I$ is the unit $2 \times 2$ matrix. For $\omega=0, b^{2}=0$, and for $\omega \neq 0, a=\sinh ^{2} \omega$.

\section{Appendix A}

\section{Identical Algebras}

Let us consider a composition class $\mathscr{J}$ whose elements are generated by a single algebra $\mathfrak{U}=\{\mathscr{H}, \sigma, \alpha\}$ :

$$
\mathscr{J}=\{\mathscr{F}, \mathfrak{A}, \mathfrak{H} \bigcirc \mathfrak{A}, \ldots\} .
$$

The underlying linear space of the algebras in $\mathscr{J}$ are tensor powers of $\mathscr{H}$ :

$$
\mathscr{H}^{n} \equiv \mathscr{H} \otimes \mathscr{H} \otimes \ldots \otimes \mathscr{H} .
$$

Since there exist two other tensor powers, the totally symmetric power

$$
\mathscr{H}^{(n)} \equiv \mathscr{H} \vee \mathscr{H} \vee \ldots \vee \mathscr{H}
$$

and the totally antisymmetric power

$$
\mathscr{H}^{[n]} \equiv \mathscr{H} \wedge \mathscr{H} \wedge \ldots \wedge \mathscr{H},
$$

it is natural to inquire whether they can be used as underlying linear spaces of composable two-product algebras.

The answer is affirmative in the symmetric case, i.e. there exist composition classes of the type

$$
\mathscr{J}=\left\{\mathscr{F}, \mathfrak{U}, \mathfrak{X}^{(2)}, \ldots\right\} .
$$

This is the standard composition of observables belonging to identical systems in quantum mechanics and in statistical classical mechanics. The antisymmetric powers are not closed under the algebraic products $\sigma, \alpha$, i.e. if $f, g \in \mathscr{H}^{[n]}$, then $f \sigma g$ and $f \alpha g$ are not in $\mathscr{H}^{[n]}$ for $n>1$. Hence, there is no composition class based 
on the antisymmetric powers. However, it is interesting to note that, for each $n$, the linear space

$$
\mathscr{H}^{\{n\}} \equiv \mathscr{H}^{(n)} \oplus \mathscr{H}^{[n]}
$$

with the composition-induced products $\sigma, \alpha$ is a two-product algebra $\mathfrak{A}^{\{n\}}$. Composition in the class

$$
\mathscr{J}=\left\{\mathscr{F}, \mathfrak{A}, \mathfrak{U}^{\{2\}}, \ldots\right\}
$$

is then based on the product $\square$ defined by the relation

$$
\mathscr{H}^{\{n\}} \square \mathscr{H}^{\{m\}} \equiv \mathscr{H}^{(n+m)} \oplus \mathscr{H}^{[n+m]} .
$$

We now outline the proofs of the above statements. A general element in $\mathscr{H}^{(n)}$ or $\mathscr{H}^{[n]}$ is a sum of elements of the form

$$
f=C^{i_{1} \ldots i_{n}} f_{i_{1} \ldots f_{i_{n}}},
$$

where summation over all indices $i=1, \ldots, n$ is understood, and where, as before, juxtaposition of variables is to be understood as their tensor product. If $f \in \mathscr{H}^{[n]}$, we have $C^{i_{1} \ldots i_{n}}=\varepsilon^{i_{1} \ldots i_{n}}$, where $\varepsilon^{\cdots}$ is the completely antisymmetric symbol in $n$ variables. If $f \in \mathscr{H}^{(n)}, C^{i_{1} \ldots i_{n}}=\left|\varepsilon^{i_{1} \ldots i_{n}}\right|$.

The elements $f \sigma g$ and $f \alpha g$ are obtained most easily by computing $f \beta g$ where $\beta=\sigma+b \alpha$, with $b^{2}=-a$ in the quantal case, while in the classical case $b$ is a nilpotent, $b^{2}=0$. With $g=D^{j_{1} \ldots j_{n}} g_{j_{1}} \ldots g_{j_{n}}$, one has

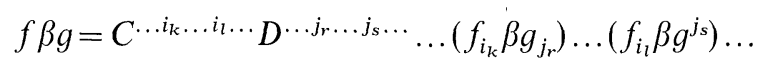

which is a sum of $n !$ terms of the form

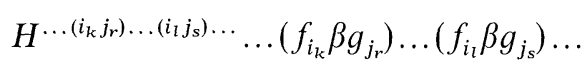

where single indices are now represented by pairs of indices.

In the symmetric case, $\mathscr{H}^{(n)}$, a transposition of two indices leaves the coefficients $C^{\cdots}$ unchanged, while, in the antisymmetric case, $\mathscr{H}^{[n]}$, it multiplies them by -1 . Thus, one can write

$$
C^{\ldots i_{k} \ldots i_{1} \ldots}=c C^{\ldots i_{1} \ldots i_{k} \ldots}
$$

where $c= \pm 1$, and similarly for $D^{\cdots}$ and $H^{\cdots}$. One then obtains from the above expressions the equation

$$
c d=h
$$

which proves the assertions.

\section{Appendix B}

\section{Relation between Classical and Quantal Algebras}

The parameter $a$, which is an element of structure in a classical or quantal algebra $\{\mathscr{H}, \sigma, \alpha\}$, appears also in the composition law for the product $\sigma$. Thus, the com- 
position laws (8a), (8c), may contain information regarding the relation between classical and quantal algebras.

Relations (8a) and (8c) are reminiscent of the addition formulas for the sine and cosine functions, and hence it might be instructive to express the operators $\sigma$ and $\alpha$ as the cosine and the sine of an "angle" operator. Since the domain $\mathscr{H} \otimes \mathscr{H}$ of $\sigma$ and $\alpha$ is different from their range $\mathscr{H}$, they cannot themselves be expressed as formal power series. However, a power series expansion can be made after a suitable factorization of the "angle" operator. Specifically, let $\alpha^{\prime}, \sigma^{\prime}, P$ be three linear operators such that the diagram

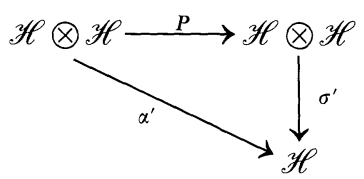

is commutative, i.e. $\alpha^{\prime}=\sigma^{\prime} \circ P$. The maps $\sigma^{\prime}$ and $\alpha^{\prime}$ are products in $\mathscr{H}$, and since the map $P$ is a linear endomorphism, one can obtain new products $\gamma$ in $\mathscr{H}$ from $P$ and $\sigma^{\prime}$ of the form $\gamma=\sigma^{\prime} \circ F(P)$, where $F(P)$ is a power series in the operator $P$.

The formal similarity between the composition laws and the trigonometric addition theorems suggests writing the products $\sigma$ and $\alpha$ in a quantal algebra $\{\mathscr{H}, \sigma, \alpha\}$ over the field of real numbers in the form

$$
\begin{aligned}
& \sigma=\sigma^{\prime} \circ \cos (\sqrt{a} P) \\
& \alpha=\sigma^{\prime} \circ(1 / \sqrt{a}) \sin (\sqrt{a} P) .
\end{aligned}
$$

When the parameter $a \in \mathbb{R}$ approaches zero, the operators $\sigma$ and $\alpha$ approach the products $\sigma^{\prime} \circ I$ and $\sigma^{\prime} \circ P=\alpha^{\prime}$, i.e. the "classical limit" of the quantal products is the products $\sigma^{\prime}, \alpha^{\prime}$, that were used to obtain the operator $P$.

We shall show that these heuristic considerations lead to a simple proof of a result similar to that obtained by C. L. Mehta [2] concerning the relation between the classical and quantal Lie products. Let $\left\{\mathscr{H}, \sigma^{\prime}, \alpha^{\prime}\right\}$ be a classical twoproduct algebra over the field $\mathbb{R}$, with products $\sigma^{\prime}$, $\alpha^{\prime}$ which permit a factorization $\alpha^{\prime}=\sigma^{\prime} \circ P$, as in the diagram. The operator $P$, being an endomorphism of the linear space $\mathscr{H} \otimes \mathscr{H}$, can be written as $P=\sum_{A} Q^{A} \otimes Q_{A}$, where $Q^{A}$ and $Q_{A}$ are linear operators on the space $\mathscr{H}$ and its dual space $\mathscr{H}^{*}$, respectively, and where $A$ ranges over an index set $\mathscr{I}$. Assume that for each value of the index $A$ the operators $Q^{A}$ and $Q_{A}$ are surjective. We shall show that under these conditions the structure

$$
\left\{\mathscr{H}, \sigma^{\prime} \circ \cos (\sqrt{a} P), \sigma \circ(1 / \sqrt{a}) \sin (\sqrt{a} P)\right\}
$$

is a quantal algebra and is the only quantal algebra obtainable from the given classical algebra $\left\{\mathscr{H}, \sigma^{\prime}, \alpha^{\prime}\right\}$ by means of a power series expansion.

In terms of the decomposition of the operator $P$ one has

$$
f \alpha^{\prime} g=\sigma^{\prime} \circ P(f \otimes g)=\sigma^{\prime} \circ \sum_{A}\left(Q^{A} f\right) \otimes\left(Q_{A} g\right)=\sum_{A}\left(Q^{A} f\right) \sigma^{\prime}\left(Q_{A} g\right) .
$$


The derivation rule for $\alpha^{\prime}$ with respect to $\sigma^{\prime}$ assumes the form

$$
\sum_{A}\left(Q^{A} f\right) \sigma^{\prime}\left(Q_{A}\left(g \sigma^{\prime} h\right)\right)=\sum_{A}\left\{\left(Q^{A} f\right) \sigma^{\prime}\left(Q_{A} g\right) \sigma^{\prime} h-g \sigma^{\prime}\left(Q^{A} f\right) \sigma^{\prime}\left(Q_{A} h\right)\right\}
$$

or

$$
\sum_{A}\left(Q^{A} f\right) \sigma^{\prime}\left\{Q_{A}\left(g \sigma^{\prime} h\right)-\left(Q_{A} g\right) \sigma^{\prime} h-g \sigma^{\prime}\left(Q_{A} h\right)\right\}=0 .
$$

Since the operator $Q^{A}$ is assumed to be surjective for each index $A$, i.e. $Q^{A} f$ is arbitrary, the expression in the curly bracket must vanish. Thus, for each $A$, the operator $Q_{A}$ is a derivation with respect to the product $\sigma^{\prime}$ :

$$
Q_{A}\left(g \sigma^{\prime} h\right)=\left(Q_{A} g\right) \sigma^{\prime} h+g \sigma^{\prime}\left(Q_{A} h\right) .
$$

A similar result holds for each operator $Q^{A}$.

For any quantal algebra $\{\mathscr{H}, \sigma, \alpha\}$ there exists a unique associative product $\beta$ with respect to which $\sigma=\frac{1}{2}[,]_{+}$and $\alpha=(1 / 2 \sqrt{-a})[,]_{-}$. Hence, we can find the quantal algebras obtainable from the given classical algebra $\left\{\mathscr{H}, \sigma^{\prime}, \alpha^{\prime}\right\}$ by determining all power series in the operator $P$ which give rise to an associative product

$$
\beta=\sigma^{\prime} \circ F(P)=\sigma^{\prime} \circ \sum_{n} C_{n} P^{n} .
$$

One sees that

$$
P^{2}(f \otimes g)=P\left(\left(Q^{A} f\right) \otimes\left(Q_{A} g\right)\right)=\left(Q^{A B} f\right) \otimes\left(Q_{A B} g\right)
$$

etc., where summation is understood and where $Q^{A B}=Q^{A}{ }^{\circ} Q^{B}=Q^{B} \circ Q^{A}$. We shall simplify the notation by writing $\left(Q^{n} f\right) \otimes\left(Q_{n} g\right)$ instead of $\left(Q^{A_{1} \ldots A_{n}} f\right) \otimes\left(Q_{A_{1} \ldots A_{n}} g\right)$. Hence $P^{n}(f \otimes g)=\left(Q^{n} f\right) \otimes\left(Q_{n} g\right)$, and

$$
f \beta g=\sum_{n} C_{n}\left(Q^{n} f\right) \sigma^{\prime}\left(Q_{n} g\right) .
$$

From the derivation rule (21) and relation (22) one obtains

$$
\begin{aligned}
& (f \beta g) \beta h=\sum_{m, n, r} C_{m} C_{n}\left(\begin{array}{l}
m \\
r
\end{array}\right)\left(Q^{m+n-r} f\right) \sigma^{\prime}\left(Q_{n} Q^{r} g\right) \sigma^{\prime}\left(Q_{m} h\right) \\
& f \beta(g \beta h)=\sum_{a, b, c} C_{a} C_{b}\left(\begin{array}{l}
b \\
c
\end{array}\right)\left(Q^{b} f\right) \sigma^{\prime}\left(Q^{a} Q_{b-c} g\right) \sigma^{\prime}\left(Q_{a+c} h\right) .
\end{aligned}
$$

Comparison of these expressions yields the following conditions on the indices:

$$
m+n-r=b, r=a, n=b-c, m=a+c
$$

which have the solution $a=r, b=m+n-r, c=m-r$. Hence, the coefficients $C_{n}$ satisfy the equations

$$
C_{m} C_{n}\left(\begin{array}{l}
m \\
r
\end{array}\right)=C_{r} C_{m+n-r}\left(\begin{array}{c}
m+n-r \\
m-r
\end{array}\right)
$$


whose solutions are $C_{n}=C / n$ ! for arbitrary $C$. Thus, $F(P)=\exp (C P)$. For the parameter $a>0$ and $C=i \sqrt{a}$, one has the algebra (20). For $a<0$ and $C=\sqrt{a}$, the circular functions are replaced by hyperbolic functions. This completes the proof.

\section{References}

1. An investigation of two-product algebras, based on the duality between observables and generators in classical and quantum mechanics, is presented in E. Grgin and A.Petersen. J. Math. Phys. 15, $764-769$ (1974)

2. Mehta,C.L.: J. Math. Phys. 5, 677-686 (1964)

3. See, e.g. Schafer, R.D.: An introduction to nonassociative algebras. Ch. V, section 3. New York: Academic Press 1966

Communicated by R. Haag

Received July 14, 1975 